\title{
EFEITOS DE ÉPOCA E NÚMERO DE APLICAÇÕES FOLIARES DE BENOMIL SOBRE A OUALIDADE DA SEMENTE DE SOJA
}

ADEMIR A. HENNING ${ }^{1}$ E WOODROW W. HARE ${ }^{2}$

RESUMO. Os efeitos da época e do número de aplicações foliares de Benomil sobre a qualidade das sementes foram avaliados através de um experimento de campo, em blocos casualizados. Os tratamentos foram: a) testemunha - sem fungicida; b) normal - duas aplicações de Benomil, a primeira no início de formação das vagens e a segunda, quatorze dias após; c) normal +1 , as duas aplicações do calendário normal, acrescidas de uma terceira, quatorze dias após a última aplicação; d) atrasada 1, as duas aplicações normais, mas com quatorze dias de atraso em relação ao calendário normal e e) atrasada 2 , idem anterior, mas iniciadas com 28 dias de atraso. Observou-se maior retenção foliar e melhor controle de doenças foliares nos tratamentos $\mathrm{c}, \mathrm{b}$ e $\mathrm{d}$ pela ordem, sendo que o tratamento e não diferiu da testemunha. $O$ peso de 250 sementes foi significativamente aumentado para todas as aplicações, todavia o maior peso de sementes foi obtido no tratamento c, sendo que os resultados dos tratamentos b, $d$ e e não diferiram entre si. As aplicações de fungicidas resultaram em um aumento significativo no vigor, porém os aumentos verificados no poder germinativo não foram estatisticamente diferentes da testemunha. A análise sanitária das sementes demonstrou que o organismo predominante foi Diaporthe phaseolorum var. sojae (Phomopsis sp) (21\%), seguido por Cercospora kikuchii $(8,4 \%)$, Aspergillus sp. (2,8\%), Fusarium sp. (1,4\%) e Miscelânea $(6,8 \%)$. Os níveis de sementes infectadas nos tratamentos com fungicida foram menores, mas não diferiram significativamente do tratamento testemunha.

Termos para indexação: fungicidas, retenção foliar, peso de 250 sementes, vigor, Phomopsis.

1 Pesquisador da EMBRAPA - Centro Nacional de Pesquisa de Soja. Caixa Postal 1061 86.100 - Londrina, PR.

2 Ph.D. Professor e Chefe do Departamento de Fitopatologia e Ervas Daninhas, da Universidade Estadual do Mississippi. P.O. Drawer P. G. Mississippi State, Miss., 39.762 - USA. 


\section{EFFECTS OF SPRAY TIMING AND NUMBER OF BENOMYL APPLICATIONS ON SOYBEAN SEED QUALITY}

ABSTRACT. The effects of spray timing and number of benomyl applications on the seed quality was evaluated through a field experiment, in a randomized comple. te block design. Treatments were: a) check, no fungicide; b) normal, two applica. tions, the first at the initial pod set and the second two weeks later; c) normal +1 , a third application added to the normal schedule, fourteen days after the last application; d) delayed 1 , the normal schedule delayed two weeks; e) delayed four weeks. Greater leaf retention and better control of foliar diseases was observed in treatments $\mathrm{c}, \mathrm{b}$ and $\mathrm{d}$ in order. Treatment e did not difter from the check The 250 seed weight was significantly increased by all fungicide treatments, howe. ver better results were obtained in treatment $c$. Treatments $b, d$ and e were not different from each other. The fungicidal applications resulted in significant increases in vigor but the increases in the germination percentage were not significant. The fungi bioassay tests revealed that Diaporthe phaseolorum var. sojae was the predominant organism within seeds. Followed by Cercospora kikuchii (8.4\%). Aspergillus sp. (2.8\%), Fusarium sp. (1.4\%) and Miscellaneous (6.8\%). The levels of infected seeds in the fungicidal treatments were lower but they were not statistically different from the control.

Index terms: fungicides, foliar retention, 250 - seed weight, vigor, Phomopsis.

\section{INTRODUC̣ÃO}

No campo, as plantas de soja estão expostas a inúmeros patógenos capazes de reduzir o rendimento e a qualidade das sementes. A extensão das perdas dependerá do tipo de patógeno, do estádio de desenvolvimento das plantas, da severidade da doença em plantas individuais e do número de plantas atacadas.

Sementes de baixa qualidade normalmente são enrugadas, descoloridas e de baixa germinação. Além de determinadas condições físicas, como clima úmido e quente durante as três últimas semanas de maturação, alguns microrganismos são agentes importantes e responsáveis pela redução na qualidade da semente.

Segundo Wyllie (16), os efeitos desses patógenos podem ser tanto diretos sobre a vagem ou a semente, quanto indiretos, afetando os processos vitais necessários à produção de sementes de boa qualidade.

O atraso na colheita é, talvez, o fator mais importante na deterioração da 
qualidade da semente, segundo Ellis e Sinclair (6) e Nangyu (10). Atow e Laviolette (1), em 1973, e Kilpatrick (9), em 1957 relataram que a percentagem de sementes infectadas era mínima antes da maturação e que esta aumentava gradativamente durante e após a maturação.

Wilcox et al (14), em 1974, observaram que a emergência das plântulas era significativamente reduzida quando a colheita foi adiada por oito a dez semanas. Ao mesmo tempo, observaram aumento significativo no número de sementes infectadas por Diaporthe phaseolorum var. sojae e Alternaria spp., não ocorrendo o mesmo com relação a Cercospora kikuchii.

O controle das diversas doenças de vagem, haste e folhas tem sido feito através do desenvolvimento de cultivares geneticamente resistentes e também por algumas práticas culturais.

Mais recentemente, a aplicação de fungicidas a nível experimental tem, algumas vezes, resultado em aumentos no rendimento, conforme trabalhos de inúmeros autores $(2,3,4,7,8,12,15)$. Por outro lado, existem diversos trabalhos relatando que a aplicação foliar de fungicidas pode ocasionar melhoria na qualidade da semente produzida $(3,5,6,11,12)$.

Ellis et al (5), em 1974, realizaram um experimento testando diferentes doses e épocas de aplicação de benomil. Segundo eles, uma única aplicação de benomil entre os estádios de floração e fim de formação de vagens seria suficiente para reduzir a incidência de Diaporthe, resultando na produção de semente de melhor qualidade. Resultados similares foram encontrados por Prasartsee et al (11), os quais relataram que benomil e chlorothalonil, além de melhorarem a qualidade das sementes, elevaram significativamente o peso das sementes.

Posteriormente, Ellis e Sinclair (6) relataram a eficiência dos fungicidas no controle dos patógenos das sementes, principalmente em situações de atraso da colheita. Segundo os mesmos autores, as aplicações foliares de benomil, além de proporcionarem aumentos de germinação "in vitro" e emergência de campo, poderiam controlar o estabelecimento de fungos nas sementes de soja por até 30 dias após a maturação, protegendo conseqüentemente a semente durante o armazenamento e, mesmo ainda no campo.

$\mathrm{O}$ presente trabalho foi conduzido com a finalidade de avaliar os efeitos do número e da época de aplicação de benomil sobre a qualidade fisiológica e sanitária da semente de soja.

\section{MATERIAL E MÉTODOS}

Este estudo foi conduzido em 1976, na fazenda "Norte" da Universidade Estadual do Mississippi, USA, sendo utilizada a cultivar "Forrest". 
Os efeitos da época e do número de aplicações foliares de benomil sobre a qualidade da semente foram avaliados através de um experimento de campo, em blocos completos casualizados, consistindo de cinco tratamentos com quatro repetições. Quatro fileiras de plantas de 6,1 m de comprimento, espaçadas de $1 \mathrm{~m}$, constituíram cada parcela.

Quando as parcelas do tratamento testemunha apresentavam-se quase totalmente desfolhadas (secas), as plantas foram avaliadas visualmente para a determinação do índice de retenção foliar, considerando-se a desfolha nas suas três seções: base, meio e ápice. Os resultados foram expressos em percentagem.

A qualidade das sementes foi avaliada pelo teste de germinação, crescimento de radícula e análise sanitária, sendo que estes testes foram conduzidos após seis meses de armazenamento à temperatura ambiente $( \pm 250 \mathrm{C})$.

\section{Teste de germinação}

Foram utilizados os procedimentos descritos em "Rules For Testing Seeds" (13), com a diferença de que foram utilizados quatro repetições de 50 sementes ao invés de quatro com 100 sementes. A percentagem de germinação foi determinada fazendo-se a primeira contagem no quinto dia e a final no oitavo dia. A média de plântulas foi tomada como percentagem de germinação.

\section{Crescimento de radícula}

Quatro repetições de dez sementes foram utilizadas por parcela, para a avaliação desse parâmetro de vigor. As sementes foram colocadas sobre uma linha reta através do comprimento das toalhas de papel e cobertas com uma toalha de papel umidecido. As toalhas enroladas foram colocadas em bandejas de metal a 450 de inclinação, num germinador a $25^{\circ} \mathrm{C}$. Após cinco dias, o comprimento da radícula de cada plântula foi medido em centímetros, calculando-se a média de cada repetição. Sementes não germinadas receberam valor zero.

\section{Análise sanitária das sementes}

Cento e vinte e cinco sementes por tratamento (cinco conjuntos de $25 \mathrm{se}$ mentes) foram coletadas, ao acaso, esterilizadas superficialmente com solução 0,525\% de Hipoclorito de Sódio por cinco minutos. Posteriormente as sementes foram enxaguadas quatro vezes em água destilada autoclavada. Cinco placas de Petri contendo BDA + 50 mg de 2,4-D, por litro com cinco sementes cada, foram monta. 
das para cada parcela. Após um período de incubação de cinco dias a 270 - $280 \mathrm{C}$, foi feita a identificação e contagem das colônicas de fungos, e os resultados, expressos em percentagem.

\section{Peso de 250 sementes}

Secções de $1,5 \mathrm{~m}$ da fileira central de plantas foram colhidas ao acaso em cada parcela. As vagens foram abertas manualmente e as sementes. ápós a limpeza. foram contadas e pesadas.

\section{RESULTADOS E DISCUSSÃO}

Os tratamentos foram: a) testemunha, sem fungicida: b) normal. duas apli. cações de benomil. a primeira no início de formação das vagens e a segunda. duas semanas após; c) normal +1 . uma terceira aplicacão adicionada ao calendário normal, duas semanas após a segunda aplicação: d) atrasada 1 as duas aplicaçóes do calendário normal, mas iniciadas com duas semanas de atraso e e) atrasada 2. idem anterior, mas iniciadas com quatro semanas de atraso (Tabela 1).

A retenção foliar, em plantas pulverizadas com certos fungicidas tem sido relatada por diversos autores $(2,3,7,11)$. Este fato, na opinião de alguns (3, 11) é o resultado do controle das doenças causadoras de desfolha. podendo proporcionar aumentos no rendimento simplesmente por proporcionar um periodo mais longo para o enchimento da semente (2,3,7).

Constatou-se no presente estudo. que a retenção foliar ocorreu por mais tempo em todos os tratamentos com fungicida. O tratamento com trés aplicaçōes de benomil produziu efeito mais acentuado que as duas aplicações do tratamento Normal, que por sua vez foi superior aos tratamentos Atrasada i e Atrasada 2 (Tabela 2). Possivelmente nos dois últimos tratamentos. o atraso na aplicação do fungicida possibilitou o estabelecimento e maior desenvolvimento das doenças tipicamente causadoras de desfolha. como a septoriose (mancha parda).

$O$ peso das sementes foi significativamente elevado em todos os tratamentos com fungicida e novamente o tratamento com três aplicaçôes (Normal +1 ) foi superior a todos os demais. Tais aumentos no peso das sementes podem explicar talvez em parte, os incrementos no rendimento da soja quando pulverizadas com certos fungicidas, uma vez que o peso da semente é um dos componentes da produção (Tabela 3). 
Tabela 1. Esquema de pulverização de benomil sobre soja, visando a avaliação dos seus efeitos sobre a qualidade da semente.

Tratamentos

Semanas após a início de formação das vagens

\begin{tabular}{llll}
0 & 2 & 4 & 6 \\
\hline
\end{tabular}

Testemunha

Normal

$1 / /\left.\right|^{1}$

$1 / 1 /$

Normal +1

$1 / 1 /$

$1 / 11$

$1 / 1 /$

Atrasada 1

$-$

$1 / 1 /$

$1 / 11$

Atrasada 2

$-$

$1 / 1 /$

Pulverização.

Tabela 2. Percentagem de desfolhamento de soja, cv. Forrest. submetida à pulverização foliar com benomil em diferentes épocas, considerando os terços inferior, médio e superior das plantas.

Tratamentos

Localização das folhas nas plantas

\begin{tabular}{lccc}
\cline { 2 - 3 } & Base & Meio & Ápice \\
\hline Testemunha & 100,0 & $100,0 \mathrm{~b}^{1}$ & $98,7 \mathrm{~d}$ \\
Normal & 100,0 & $85,0 \mathrm{~b}$ & $72,5 \mathrm{~b}$ \\
Normal + 1 & 100,0 & $75,0 \mathrm{a}$ & $62,5 \mathrm{a}$ \\
Atrasada 1 & 100,0 & $95,0 \mathrm{c}$ & $88,7 \mathrm{c}$ \\
Atrasada 2 & 100,0 & $98,7 \mathrm{~cd}$ & $93,7 \mathrm{~cd}$
\end{tabular}

${ }^{1}$ Dentro de cada coluna, duas médias seguidas da mesma letra não diferem significativamente ao nível de $5 \%$ de probabilidade, conforme determinado pelo teste de Duncan. 
Tabela 3. Peso de 250 sementes de soja, cv. Forrest, submetida à pulverização foliar com benomil em diferentes épocas, e respectivos aumentos percentuais em relação à testemunha sem tratamento.

\section{Tratamentos}

Testemunha

Normal

Normal +1

Atrasada 1

Atrasada 2
Peso de 250 sementes (g)
Aumento

(\%)

\section{$27,75 c^{1}$}

$31,53 \mathrm{~b}$

32,91 a

$31,28 \mathrm{~b}$

$31,47 \mathrm{~b}$
13,62

18,60

12,72

13,40

${ }^{1}$ Duas médias acompanhadas pela mesma letra não diferem significativamente entre si ao nível de $5 \%$, conforme determinado pelo teste de Duncan.

Cada aplicação de benomil foi feita na dose de $560 \mathrm{~g}$ ia/187 $\ell$ água/ha.

Devido ao clima úmido e ao acamamento de algumas plantas, utilizou-se uma barra de pulverização manual, com cinco bicos 8001 -E, à pressão de 40 libras por polegada quadrada $\left(2,81 \mathrm{~kg} / \mathrm{cm}^{2}\right)$, fornecida por um tanque de $\mathrm{CO}_{2}$.

Provavelmente, o longo período de armazenamento à temperatura ambiente afetou a germinação, fazendo com que os aumentos observados não fossem significativos (Tabela 4). Já com relação ao vigor, todos os tratamentos com fungicida foram superiores ao tratamento testemunha, não havendo diferença estatística entre épocas e número de aplicações. Todavia, o atraso de quatro semanas na aplicação dos fungicidas, prejudicou um pouco o vigor das sementes.

Phomopsis sp. e Cercospora kikuchii foram os dois microrganismos predominantes nas sementes. No tratamento testemunha, $42 \%$ das sementes estavam infectadas com Phomopsis sp., ao passo que em nenhum dos outros tratamentos este valor foi superior a 19\% (Tabela 5). Apesar da diferença entre os tratamentos não, ser estatisticamente significativa, observou-se que todos os tratamentos com fungicida reduziram acentuadamente este índice, que foi de apenas $12 \%$ no tratamento com três aplicações de benomil. 
Resumindo, em termos de qualidade de semente, não se encontrou vantagem em se atrasar o calendário normal, nem tampouco, em se adicionar uma terceira pulverização. Alguns parâmetros, como o peso đas sementes e a incidência de pató. genos nas sementes, foram beneficiados por tais práticas. No entanto, a germinação e o vigor se mostraram superiores no tratamento Normal.

Tabela 4. Percentagens de germinação e de vigor de sementes de soja, cv. Forrest, armazendas por período de seis meses à temperatura ambiente, e obtidas de plantas pulverizadas com benomil em diferentes épocas.

Tratamentos

Testemunha

Normal

Normal +1

Atrasada 1

Atrasada 2

\section{Germinação}

(\%)
$\%$ de sementes com alto vigor
57,25

77,37

76,32

74,12

68,37

$\mathrm{NS}^{1}$
$45,62 b^{2}$

$71.25 \mathrm{a}$

$68.75 \mathrm{a}$

$68,75 \mathrm{a}$

$58,75 \mathrm{a}$

Valores não significativos calculados ao nível de $5 \%$ de probabilidade.

2

Dentro da mesma coluna, duas médias acompanhadas pela mesma letra não diferem significativamente ao nível de $5 \%$ de probabilidade, conforme determinado pelo teste de Duncan. 
Tabela 5. Percentagem de sementes de soja da cv. Forrest infectadas por fungos de acordo com a época e o número de pulverizações de benomil.

\begin{tabular}{lllcccr}
\hline \multirow{2}{*}{ Organismos } & \multicolumn{5}{c}{ T r a t a m e n t o s } \\
\cline { 2 - 6 } & Testemunha & Normal & Normal + 1 & Atrasada 1 & Atrasada 2 & Média \\
\hline $\begin{array}{l}\text { D. phaseolorum } \\
\text { (Phomopsis) }\end{array}$ & 42,0 & 19,0 & 12,0 & 14,0 & 18,0 & 21,0 \\
C. kikuchii & 7,0 & 6,0 & 11,0 & 8,0 & 10,0 & 8,4 \\
A spergillus sp. & 0,0 & 6,0 & 4,0 & 4,0 & 0,0 & 2,8 \\
Fusarium sp. & 3,0 & 1,0 & 0,0 & 0,0 & 3,0 & 1,4 \\
Miscelânea & 5,0 & 6,0 & 8,0 & 5,0 & 10,0 & 6,8 \\
Total NS & 57,0 & 38,0 & 35,0 & 31,0 & 41,0 & 40,4 \\
\hline
\end{tabular}

${ }^{1}$ Percentagem total de sementes infectadas por tratamento, valores não significativos calculados ao nível de $5 \%$ de probabilidade. 


\section{REFERÊNCIAS}

01. ATOW, K. L. \& LAVIOLETTE, F. A. Pod protection effects on soybeans seed germination and infection with Diaporthe phaseolorum var. sojae and other microorganisms. Phytopathology, 63 (8): 1021-3, aug. 1973.

02. BARNETT, R. D. \& LUKE, H. H. The effects of fungicides on disease development, seed germination, and grain yield of wheat. Pl. Dis. Rep., 60 (2): 117-9, feb. 1976.

03. BOONYNG, V. Effect of foliar application of benomyl on seed quality and yield in soybeans. Mississippi State University, 1976. n.p. Tese(PhD) Mississippi State University.

04. EDGINGTON, L. U.; REINBERGS, E.; SHEPARD, H. C. Evaluation of ethirimol and benomyl for control of powdery mildew of barley. Can. J. PI. Sci., 52 (5): 693-9, 1972.

05. ELLIS, M. A.; ILYAS, M. B.; TENNE, F. D.; SINCLAIR, J. B.; PALM, H. L. Effect of foliar applications of benomyl on internally seed borne fungi and pod and stem blight in soybeans. Pl. Dis. Rep., 58 (8): 760-3, aug. 1974.

06. — \& SINCLAIR, J. B. Effect of benomyl field aprays on internallyborne fungi, germination, and emergence of late-harvested soybeans seeds. Phytopathology, 66 (5): 680-2, may. 1976.

07. HORN, N. L.; LEE, F. N.; CARVER, R. B. Effects of fungicides and phathogens on yields of soybeans. Pl. Dis. Rep., 59 (9): 724-8, sep. 1975.

08. JEFFERS, P. L. \& SHMITTHENNER, A. F. Soybeans seed quality and yield as affected by potassium nutrition and benomyl applications. Agron. Abstr., 68 : 94, 1976.

09. KILPATRIC, R. A. Fungi associated with the flowers, pods and seeds of soybeans. Phytopathology, 47 (3): 131-5, mar. 1957. 
10. NANGJU, D. Effect of harvest time on seed quality and viability of soybeans in the tropics. Agron. Abstr., $68: 39,1976$.

11. PRASARTSEE, C.; TENNE, F. O.; ILYAS, M. B.; ELLIS, M. A.; SINCLAIR, J. B. Reduction of internally seed borne Diaporthe phaseolorum var. sojae by fungicide sprays. PI. Dis. Rep., 59 (1): 20-3, jan. 1975.

12. ROSS, J. P. Effect of overhead irrigation and benomyl sprays on late-season foliar diseases, seed infection, and yields of soybean. PI. Dis. Rep., 59 (10): 809-13, oct. 1975.

13. RULES for testing seeds. Proc. Assoc. Off. Seed Anal., 60 (2): 1-116, 1970.

14. WILCOX, J. R.; LAVIOLETTE, F. A.; ATOW, K. L. Deterioration of soybean seed quality associated with delayed harvest. PI. Dis. Rep., 58 (2): 130-3, feb. 1974.

15. WINN, J. Fungicide treatments increase soybean yields. Texas Agric. Progress, 21 (3): 18-9, 1975.

16. WYLLIE, T. D. Piant diseases and soybean production. Soybean Digest, 27 (10): 16-20, 1967. 